\title{
Towards an Intelligent Wheelchair System for Users With Cerebral Palsy
}

\author{
Luis Montesano, Marta Díaz, Sonu Bhaskar, and Javier Minguez
}

\begin{abstract}
This paper describes and evaluates an intelligent wheelchair, adapted for users with cognitive disabilities and mobility impairment. The study focuses on patients with cerebral palsy, one of the most common disorders affecting muscle control and coordination, thereby impairing movement. The wheelchair concept is an assistive device that allows the user to select arbitrary local destinations through a tactile screen interface. The device incorporates an automatic navigation system that drives the vehicle, avoiding obstacles even in unknown and dynamic scenarios. It provides the user with a high degree of autonomy, independent from a particular environment, i.e., not restricted to predefined conditions. To evaluate the rehabilitation device, a study was carried out with four subjects with cognitive impairments, between 11 and 16 years of age. They were first trained so as to get acquainted with the tactile interface and then were recruited to drive the wheelchair. Based on the experience with the subjects, an extensive evaluation of the intelligent wheelchair was provided from two perspectives: 1) based on the technical performance of the entire system and its components and 2) based on the behavior of the user (execution analysis, activity analysis, and competence analysis). The results indicated that the intelligent wheelchair effectively provided mobility and autonomy to the target population.
\end{abstract}

Index Terms-Cerebral Palsy, intelligent wheelchairs, tactile interface.

\section{INTRODUCTION}

$\mathbf{E}$ LECTRICALLY powered wheelchairs have been recognized as a primary mobility aid for the elderly as well as the physically impaired [1]. A survey on the adequacy of electric wheelchairs [2] shows that, according to clinicians, $40 \%$ of users have difficulties in steering and maneuvering in daily life environments. These scenarios include situations in which the maneuvering space is limited, the approach to furniture and objects is tightly constrained, and the necessity to pass through doorways requires precise control. The survey also shows that

Manuscript received August 16, 2008; revised August 31, 2009; accepted September 11, 2009. First published January 12, 2010; current version published April 21, 2010. This work was supported in part by the Spanish MEC projects ADA (DPI2006-15630-C02-01 and DPI2006-15630-C02-02) and in part by FCT (ISR/IST pluriannual funding) through the POS_Conhecimento Program that includes FEDER funds.

L. Montesano, S. Bhaskar, and J. Minguez are with the I3A and Department de Informática e Ingeniería de Sistemas, Universidad de Zaragoza, 50018 Zaragoza, Spain (e-mail: montesano@unizar.es; sbhaskar@unizar.es; jminguez@unizar.es).

M. Díaz is with the 4all-L@b/Research Center for Dependency Care and Autonomous Living, UPC, 08800 Vilanova i la Geltrú, Spain (e-mail: marta. diaz@upc.edu).

Color versions of one or more of the figures in this paper are available online at http://ieeexplore.ieee.org.

Digital Object Identifier 10.1109/TNSRE.2009.2039592 conventional navigation with joysticks can cause fatigue during long operational periods. Clinicians pointed out that approximately $50 \%$ of the users that are unable to control a powered wheelchair by conventional methods would benefit from automated navigation systems. In this context, intelligent wheelchairs are especially suitable for users with severe motor and/or cognitive impairments, who experience difficulties in driving standard powered wheelchairs [3]. The motivation behind these wheelchairs is to facilitate assistance in mobility in order to accomplish complex navigational tasks.

In many cases, motor disabilities come associated with cognitive and sensorial impairment (e.g., cerebral ischemia). Moreover, cognitive disabilities can lead to driving/navigational problems even when motor impairments are not severe. For users with cognitive disabilities, the survey in [2] shows that $91 \%$ of the clinicians believe that these robotic wheelchairs with automated navigation systems can be useful at least for a few users, and $23 \%$ believe the systems can be useful for many of them. It has also been pointed out that powered mobility can have tremendous positive psycho-social effects on users. In addition to independence and self-esteem, the possibility of enhanced self-locomotion provided by a wheelchair also has special beneficial effects in regard to the development and rehabilitation of children with disabilities [4]. A more recent study [5] analyzed the potential user population for smart wheelchairs, including those with autonomous navigation capabilities. They argued that a large number of wheelchair users can benefit from this type of wheelchairs. Although research has been carried out in the area of intelligent wheelchairs (see [3], [6]-[8] for reviews), very little recent work has been devoted to understand the applicability of this technology for users with cognitive disabilities [9], [10].

This paper describes an intelligent wheelchair, adapted for users with cognitive disabilities, as well as presents a field study to evaluate the wheelchair. The study focuses on users with cerebral palsy, one of the most common disorders that affects muscle control and coordination impairing movement.

The paper is organized as follows. Section II presents the methods used in the study, which include 1) the design of the intelligent wheelchair (i.e., the wheelchair platform, the user interface and the intelligent navigation system), 2) the selection of the participants, 3) the design of the experiments, and 4) the definition of the evaluation metrics. Section III analyzes the results based on the previous set of metrics. Section IV presents the conclusions of the study. 


\section{METHODS}

\section{A. Intelligent Wheelchair}

The starting point is a commercial electric wheelchair that complies with the basic ergonomic requirements and also with the mobility of the users. The wheelchair is equipped with the computational, sensory, and control resources required to implement the full system architecture. Two Intel 800-Mhz computers are installed on board; one for low-level control (real-time operative system, VxWorks) and the other to run the navigation system and user interface (Windows). This computer also manages the input/output devices (sound and tactile VGA screen). Both computers are connected to RS-232 and Ethernet. The low-level computer controls the rear wheels, working in a differential-drive mode. The main sensor is a SICK LMS200 planar laser, placed at the front part of the vehicle, at a height of $0.75 \mathrm{~m}$. The laser operates at $5 \mathrm{~Hz}$, with a $180^{\circ}$ field-of-view and a $0.5^{\circ}$ resolution (361 points). This sensor provides information about the obstacles in front of the vehicle. The wheelchair is also equipped with wheel encoders (odometry), and a wireless Ethernet card that allows connecting the vehicle to a local network during operation.

\section{B. User-Machine Shared Control Support}

One of the primary issues to be considered in any intelligent wheelchair is the user autonomy support, which fixes the type and degree of autonomy of the user for controlling the wheelchair. As the wheelchair will also make its own decisions, it is necessary to establish a control strategy between the user and the system (see [11] for a taxonomy). The most widespread mode for robotic wheelchairs is shared control, which focuses on combining the instructions of the operator with the robot's assessment of the environment. Although this mode has been widely used for wheelchairs (e.g., [12]-[19]), its applicability to subjects with cognitive disabilities has not been explored to date. Among all possible shared control strategies, the final destination is chosen, where the user selects arbitrary local destinations and the wheelchair autonomously navigates to the desired locations. In other words, the user is responsible for the high level planning, either by selecting intermediate goals to reach a desired location, or by simply exploring the environment. The wheelchair autonomously navigates with safety among these intermediate goals, considering the low level control of the vehicle. This strategy minimizes the user input as suggested by recent studies [20], and the machine deals with all aspects of navigation.

\section{User Interface}

Within the area of assistive robotic wheelchairs, much work has been accomplished in human-machine interfaces, as it is critical in determining the performance and acceptance of the device by the user [21]. For instance, there are tactile devices [17], voice devices [22], [23], graphical interfaces [12], [14], [15], [22]-[24], joysticks [14], [16], [19], [22], gaze tracking or air expulsion [22], brain computer interfaces [18], [25], facial gestures [26], to name a few. However, very few works have addressed the usability for users with cognitive disabilities. For

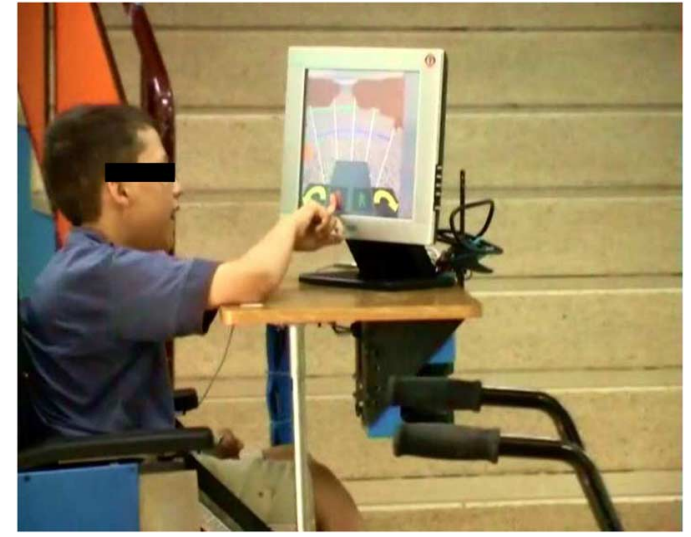

Fig. 1. Snapshot of a subject selecting a command on the visual display by touching the screen.

example, [27] has recently evaluated an interface for users with cognitive impairments, in the context of a robotic arm mounted on a wheelchair; virtual reality interfaces have been reported to be a valuable tool in brain damage rehabilitation [28], and in the assessment of the driving skills of users with cognitive disabilities [29], [30]. The use of virtual environment allows the comparison of alternative control methods under simulated repeatable conditions which is difficult in real applications.

1) Touchscreen as Input Device: This study focuses on users who have the motion of one arm relatively intact and no visual impairments. A touchscreen is selected as it provides an integrated version of both the physical device (to interact with the machine) and the user display (Fig. 1). Its advantage is the ease of usage and robustness, when compared to other interfaces such as voice recognizers [10]. The screen is located on the wheelchair table but displaced to the left-hand side (or right-hand side depending on the laterality of the user) in order to maintain the user's frontal view free/unobstructed.

To command the wheelchair, the user has to be able to select intermediate destinations and to stop it at any time by touching on the screen. Thus, the screen displays information on the vehicle's current assessment of the environment and also additional information required for command selection (Fig. 2). Furthermore, based on this information, the user selects commands or options by directly touching the screen, following a given operation protocol.

2) Visual Display: Human factor studies on interfaces suggest that efficiency is highly linked to situation awareness, as the lack of awareness increases workload and errors [31], [32]. In this case, the effect is even more pronounced because low situation awareness leads to comprehension problems (this was evident in the first visual display prototype [10]). To ameliorate the situation awareness, sensor fusion [33], [34] is used to create a 3-D real-time reconstruction of the environment [35]. More precisely, the visual interface shows a 3-D virtual world model constructed online, as would be seen from a virtual camera located approximately at the user's eyes. Furthermore, the user selects the commands directly on the 3-D model on the screen, avoiding the use of menu-based systems, in accordance with the results of [20]. The design of the visual display is based on previous experience with other representations for children with cognitive 


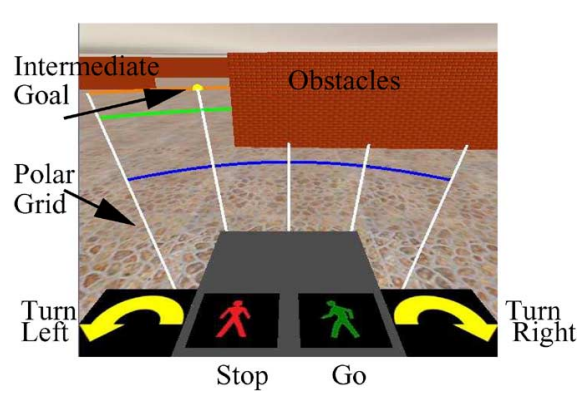

Fig. 2. Representation of the visual display: the obstacles are depicted by walls, the grey cube represents the wheelchair, the grid over the floor maps the possible goal locations, the arrow buttons turn the vehicle from its current position, and the traffic light buttons validate or reject the user's commands.

disbilities [10], in which it was observed that 3-D abstractions of the scenario had to be built.

More precisely, the visual display is a reconstruction of the scenario and presents additional information to help the user select a command (Fig. 2). The 3-D environment visualization is built from the 2-D map constructed in real time by the autonomous navigation technology (see next section). The use of an online map instead of a priori map increases the versatility of the application, as this strategy works on unknown scenarios and rapidly reflects changes in the environment, such as moving people or unpredictable obstacles, like tables and chairs. To assist the user's situation awareness, the wheelchair is co-located within the map and both items are displayed on the screen, originating from a virtual camera located beside the user's eyes. In other words, the visual information on the screen is a simplified reconstruction of the perception of the user. Although the following design might seem abstract, the users rapidly understood the mechanism and learnt to use it.

The rest of the displayed information helps the user select a command. Firstly, a set of destinations is defined, relative to the wheelchair, which are locations in the environment that the operator may select to reach with the wheelchair. The interface restricts the selection of possible intermediate goals to reachable empty space locations (Fig. 2). These locations are represented on the display by an $N \times M$ polar grid attached to the wheelchair. The intersections of the grid represent real locations in the scenario, and the grid dimension is customizable. In the experiments, a $3 \times 5$ grid is used to represent locations at $(2 \mathrm{~m}, 4 \mathrm{~m}, 8 \mathrm{~m}) \times\left(-60^{\circ},-30^{\circ}, 0^{\circ}, 30^{\circ}, 60^{\circ}\right)$ from the current wheelchair location. In addition, there are also specific available actions, represented by icons on the visual display. The first set of actions turn the wheelchair in place, to the right or to the left $\pm 90^{\circ}$. Note that these commands are essential to navigation, as the interface only shows a partial view of the environment around the wheelchair. By turning the wheelchair, the user can achieve a $360^{\circ}$ view of the environment and select goals that are currently outside the interface. The corresponding icons are located on the right- and left-hand sides of the wheelchair tetrahedron, and represented by a turning arrow in the respective directions. The second set of actions validates or cancels the previous selections. They are represented by familiar icons, located on the wheelchair.

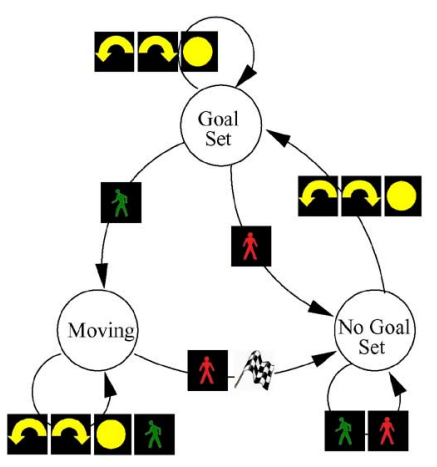

(a)

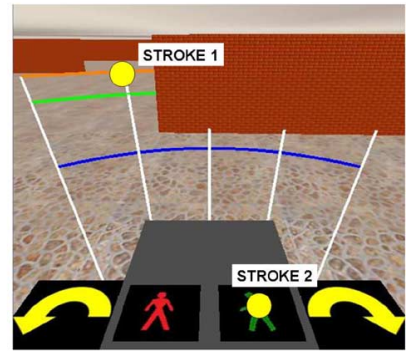

(b)
Fig. 3. (a) Finite state machine of the input protocol. (b) Example of the selection of one destination with two pulsations on the screen. Initially the state is No Goal Set. The user types a destination on the grid (stroke 1), which is displayed on the screen. The new state is Goal Set. Next, the user selects the run icon (stroke 2) and the state switches to Moving.

A yellow circle on the polar grid indicates the last selected goal, remaining active after confirmation until the vehicle reaches it. The icons on the lower part of the interface illuminate when used. Every element of the display can be customized in terms of color, shape, size, or location, according to the capabilities and preferences of the user.

The user selects the options by touching the screen. To facilitate this selection (especially under low precision arm control conditions), the visual display is divided into regions-not visible to the user-that encompass locations and buttons. A selection is made when the user touches the screen within one of these regions; an auditory signal confirms that the selection is processed.

3) Operation Protocol: It defines the way the user utilizes the options provided on the visual screen. A finite state machine models the behavior of the system [Fig. 3(a)]. Initially, the state is No goal set. When the user selects a command (either a destination or a turn), the state turns to Goal set. Then, the user validates the command with the green icon (state Moving), or rejects it with the red icon No goal set. The user may change the goal or start a turning behavior without halting the wheelchair (maintaining the Moving state), or stop the vehicle at any time (switching the state to No goal set). When the wheelchair reaches the final destination, the state changes to No goal set and the system waits for further commands. Fig. 3(b) shows an example of selecting one destination.

\section{Navigation Technology}

It is worthy to recall that the aim of this study is to develop an intelligent robotic wheelchair to provide independent mobility for people with cognitive and motor disabilities. The device should be able to operate in unknown and populated scenarios, giving the user total freedom, and avoiding restrictive operation constraints or predefined settings. Thus, the wheelchair concept does not establish predefined settings or makes assumptions about the working conditions. Although there are wheelchairs that provide navigation assistance (e.g., [13]-[16], [19], [26], to name a few), they usually constrain the operational conditions. For example, some of them assume a priori knowledge 
TABLE I

SUBJECT PROFILES

\begin{tabular}{|c|c|c|c|c|}
\hline$\overline{\text { Characteristics }}$ & Subject I & Subject II & Pubject III & Subject IV \\
\hline Sex, age, handedness & Female, 16, Left-handed & Male, 14, Left-handed & Male, 16, Right-handed & Male, 11, Left-handed \\
\hline $\begin{array}{l}\text { Motor disability and } \\
\text { Clinical symptoms }\end{array}$ & $\begin{array}{l}\text { Cerebral paralysis due to hy- } \\
\text { poxic Ischemic encephalopa- } \\
\text { thy. }\end{array}$ & $\begin{array}{l}\text { Cerebral ischemia in central } \\
\text { lobes and motor aphasia. }\end{array}$ & $\begin{array}{l}\text { Tetraparesis due to } \\
\text { encephalopathy with spastic } \\
\text { diplegia and nystasmus due } \\
\text { to cerebral paralysis. }\end{array}$ & $\begin{array}{l}\text { Cerebral paralysis with maturity } \\
\text { deficiency and reduced visual ca- } \\
\text { pabilities. }\end{array}$ \\
\hline Cognitive disability & $\begin{array}{l}\text { Moderate mental retardation } \\
\text { given the Wechsler Intelli- } \\
\text { gence Scale (oral I.Q.: 70; } \\
\text { manipulative I.Q. } 47 \text {; and } \\
\text { general I.Q. 55). }\end{array}$ & $\begin{array}{l}\text { Moderate mental retardation } \\
\text { given the Wechsler Intelli- } \\
\text { gence Scale (oral I.Q.: } 66 \text {; } \\
\text { manipulative I.Q.: } 57 \text {; and } \\
\text { general I.Q.: 55). }\end{array}$ & $\begin{array}{l}\text { Moderate mental retardation } \\
\text { given qualitative observa- } \\
\text { tions for DSM- } I V \text { parame- } \\
\text { ters. }\end{array}$ & $\begin{array}{l}\text { Moderate mental retardation } \\
\text { given qualitative observations } \\
\text { for DSM- } I V \text { parameters and } \\
\text { Wechsler Intelligence Scale (only } \\
\text { oral I.Q. available: } 80 \text {; }\end{array}$ \\
\hline
\end{tabular}

about the scenario-e.g., internal maps, landmarks or characteristic places; motion in noncomplex scenarios-e.g., in confined spaces; or motion over precomputed or previously learned motions-e.g., precomputed paths. For this reason, this study starts by relying on advanced robotic technology in mobile robotics to address navigation, overcoming the previous assumptions [36]. This navigation system is able to deal with 1) very dynamic environments (people in motion) and 2) unknown scenarios (the location of the furniture, tables, chairs, rehabilitation devices, etc., are completely unpredictable during the day).

The task of the autonomous navigation system is to drive the vehicle to a given destination while avoiding obstacles, static and dynamic, detected by the laser sensor. The goal location is provided by the user by means of the user machine interface (previous subsection). As mentioned previously, the aim is to provide mobility skills even in situations where the user is moving in an unknown environment (which prevents predefined strategies), or when the environment varies with time, e.g., people in motion or changes in furniture locations. In order to implement such a complex navigation system, it is necessary to combine several functionalities. In particular, the navigation technology integrated the following modules.

1) The Model Builder: This module integrates the sensor measurements to construct a local model of the environment and track the vehicle location. A binary occupancy grid map is chosen to model the static obstacles and the free space, and a set of extended Kalman filters is chosen to track the moving objects around the robot. The technique described in [37] and [38] is applied to correct the robot position, update the map, and detect and track the moving objects around the robot. The static map travels centered on the robot. This map has a limited size, but sufficient to present the required information to the user, as described in the previous section, and to compute the path so as to reach the selected goal.

2) The Local Planner: The local planner computes the local motion based on the hybrid combination of tactical planning and reactive collision avoidance. An efficient dynamic navigation function ( $\mathrm{D}^{*}$ Lite planner [39]) is used to compute the tactical information (i.e., main direction of motion) required to avoid cyclic motions and trap situations. This function is well suited for unknown and dynamic scenarios because it works based on the changes in the model computed by the model builder. The actual motion of the vehicle is computed using the ND technique [40], which uses a "divide and conquer" strategy, based on situ- ations and actions to simplify the collision avoidance problem. This technique has the distinct advantage that it is able to provide complex navigational tasks, such as maneuvering in the environment within constrained spaces (e.g., passage through a narrow door). In order to assist with comfortable and safe operation during navigation, the shape, kinematics, and dynamic constraints of the vehicle were incorporated using a technique adaptable for differentially-driven vehicles [36]. For further details regarding the modules, their inter-operation and synchronization issues, see [36].

\section{E. Participants}

Recruitment for participation in the study began after the protocol was approved by both the University of Zaragoza Institutional Review Board and the Alborada Primary School, a school for children with cognitive disabilities in Zaragoza. Initially, the focus was on the incorporation of subjects with cerebral palsy and varied disability profiles (degree of motor impairment, cognitive skills, and sensorial disabilities) to explore the boundaries of the rehabilitative use of the powered wheelchair prototype. The selection of the participants was made by the research team (constituted of educators, a psychologist, and the rehabilitation engineers). The subjects evaluated for powered mobility were considered candidates for the study at the school. The inclusion criteria for the recruitment of subjects were 1) cerebral palsy diagnosis, 2) minimum general I.Q. and moderate mental retardation, given qualitative observations for DSM- $I V^{1}$ parameters, 3) experience in using conventional non-powered wheelchairs, 4) knowledge of Spanish (verbal), and 5) consistent motor access appropriate for the operation of the wheelchair through the interface described in Section II-F, and 6) ability to understand the proposed tasks.

All students who met criteria (1-5) were offered participation and five subjects agreed to participate in the study. In order to evaluate criterium (6), we carried out a training phase (see Section II-F) prior to the actual wheelchair trials. One of the subjects was unable to complete the training phase, failing criterium (6). Eventually, a total of four subjects (three males and one female) aged between 11-16 years of age completed the study. Table I provides a detailed description of the participants. All participants used standard nonelectric wheelchairs in their daily life, some of them semi-autonomously, and some with assistance.

\footnotetext{
${ }^{1}$ Diagnostic and Statistical Manual of Mental Disorders.
} 
None had ever utilized an electric wheelchair before. Subjects 1 and 2 are capable of driving a powered wheelchair but do not have the necessary funds to acquire the equipment. It is unclear if subjects 3 and 4 will be able to drive an standard wheelchair. All participants and their parents provided informed consent to take part in the study. To comply with ethical issues, responsibility, reflection, and transparency were maintained during the conduction of the entire protocol, from previously described selection of participants to the study design and evaluation procedures [41].

\section{F. Experiment Design and Procedures}

The study was carried out in two phases, a training phase and an evaluation phase. The training phase comprised of training each subject in the use of the navigation interface, by means of a simulator game. This game emulated the underlying mechanisms of user interface and wheelchair navigation. With this process, the subjects learned to use the interface, which can be customized to augment the adaptability of the system to the user. The experimentation phase consisted of the user navigating along an established circuit. Various data were collected for posterior evaluation and assessment. After the sessions, the subjects were interviewed by the educators (who also took the role of assistants during the test) to know their opinions about the wheelchair system and the navigation for a posterior qualitative assessment of their experiences.

1) Training Sessions: The sessions took place in the computer room at the Alborada school. For this phase, a 3-D graphics game environment was developed on a computer workstation, simulating a virtual scenario, the main characteristics of the user machine interface, and the wheelchair control. The respondents were required to accomplish simple navigational tasks within the virtual environment. These tasks involved catching several target characters positioned in various places within the space of the virtual environment. The games introduced music and popular characters as motivation factors to make the task attractive and to encourage and reinforce the learning procedure. The gaming environment was developed to fulfill the following objectives: 1) to motivate the subjects to participate in the field trials, 2) to train the subjects to interact with the touchscreen, to familiarize with the rules of the interface dialog, and to understand the interface and its relation with the wheelchair motion, 3) to identify user interface requirements that suit their understanding, and 4) to record the attempts made by the subjects in order to personalize the touchscreen. The training took place in a single session per subject. The duration of this session varied from 45 to $60 \mathrm{~min}$ depending on the user. The training was carried out by the school therapists and engineers in integration with the usual activities in the school so as not to modify the routine.

2) Field Sessions: The evaluation sessions lasted one week and took place at the school, one week after the training sessions. In this phase, a circuit was designed in the school and the participants were asked to follow it by autonomously using the intelligent wheelchair (Fig. 4). Each subject was positioned in the wheelchair by the therapists of the school. After a few minutes habituation to the wheelchair, the circuit was verbally

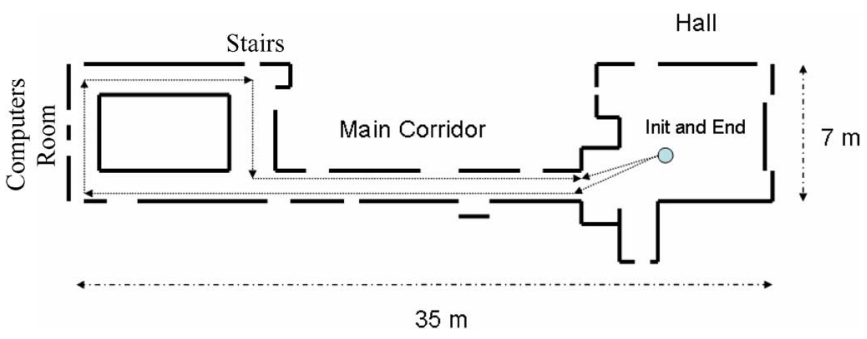

Fig. 4. Circuit followed by the children during the field trials. As the normal school operation was not modified, for the real trials there were furniture, rehabilitation devices, and people (educators and students) moving around the school.

described to the subjects, who attempted to follow it. They were asked to follow the long corridor, pass in front of the computer room and the stairs, and return to the initial location. The environment was known to the subjects.

Each subject repeated the circuit only one time during the school regular working hours, without modifying normal operation. The children's main task was to select through the interface the appropriate intermediate goals to follow the previously described global circuit. The circuit was also executed using the same protocol by one of the engineers to serve as reference and was marked as control in the results.

All trials were video recorded, as well as all data used by the wheelchair (odometry readings, laser scans, touch screen pulsations, etc.). After each trial, the participants were interviewed by the educators in order to know their opinions about the system, the interface, and to evaluate the quality of the experience. All this data are the basis of the evaluation and used to obtain the results described in the next section.

\section{G. Evaluation Metrics}

One of the objectives of this paper was to provide a quantitative evaluation of the system based on the experience with the real users. This is important not only to analyze the performance of the proposed wheelchair, but also to allow comparison with further developments and other systems. The evaluation was based on two sets of metrics: the first was related to the wheelchair evaluation as a mobility device, while the second studied the behavior of the users while using the device. Some of these metrics were incorporated from literature; others, especially those related to the behavior evaluation of the user, are innovative and are expected to constitute the first step towards a systematic evaluation of this type of systems.

1) Wheelchair Performance Metrics: Some metrics were proposed to evaluate the performance of intelligent wheelchairs [42]-[44]. Based on these metrics, three groups of metrics were established for this study.

\section{Overall performance}

- Task success: completion of the navigation task, i.e., the circuit. The presence of incidents such as collisions during the task was tolerated. A noncompleted task was considered a failure due to, for instance, user desertion, wheelchair misoperation, or failure to reach the final destination.

- Path length: Distance traveled to accomplish the task.

- Time: time Taken to accomplish the task. 
- Collisions: Number of collisions during the task. A collision is not considered a failure as long as the system is able to continue with a new command, or requires a brief intervention from the supervisor.

- Mean velocity: Mean velocity during motion (in the moving state of Fig. 3).

\section{User interface performance}

- Usability rate: Number of pulsations per mission. A pulsation is the detection of a touch. A mission is a confirmed intermediate goal or a turn command.

- Command utility: Command usage frequency.

- Device errors: Failures in the detection of input.

\section{Navigation performance}

- Mission success: Number of successful missions.

- Collisions: Number of collisions per mission, per distance and per period of time.

- Obstacle clearance: Minimum and mean distance to the obstacles.

- Robustness in narrow spaces: Number of narrow passages successfully traversed.

2) User Behavior Metrics: User behavior metrics for this type of application are rarely documented in literature. Three different but complementary points of view were focused: an execution analysis (to give insights on what the users did and their performance), an activity analysis (related to how the subjects performed the task), and a competence analysis (to give insight on what are the aptitudes and skills achieved when using the device). Together, these three studies give a measure of the degree of accomplishment and adaptability of the wheelchair to the subjects.

Execution Analysis: To measure the degree of accomplishment of the navigational task. The following metrics were proposed: 1) number of missions, 2) path length, 3) period of time taken, 4) number of collisions, and 5) number of narrow passages.

Activity Analysis: The activity analysis addresses the interaction strategy of the users with the wheelchair, in order to achieve the navigational task. Accordingly to [45], there are two types of activity that apply to this context: the supervisory oriented activity, and the direct control oriented activity. The supervisory oriented activity is defined by the lesser amount of intervention and a selection of goals that exploits the automation facilities, mainly the trajectory planning and obstacle avoidance. This mode is characterized by a higher number of pulsations towards far goal destinations, a lower number of stop, left or right arrow pulsations, and a lower number of missions. The direct control activity is characterized by an increased user intervention and less confidence in the navigation capabilities of the system. This mode is operatively described by a higher number of pulsations on the arrow icons (to orient the wheelchair), more frequent stop pulsations (to abort a trajectory), near range goal selections, and a higher number of missions.

Next, four metrics to discriminate between both interaction modalities are proposed.

- Activity discriminant: measures the ratio between destination pulsations and the number of selections (denoted by
TABLE II

GOAL ATtAINMENT AND POWERED MOBILITY SCORES

\begin{tabular}{c|ll}
\hline Score & Operational Goal Example & Functional Goal example \\
\hline \hline 0 & Troubles in using tactile screen & Task not attempted \\
\hline 1 & $\begin{array}{l}\text { Uses tactile screen with contin- } \\
\text { ual supervision }\end{array}$ & $\begin{array}{l}\text { Subject attempts task but re- } \\
\text { quires hands-on verbal and ges- } \\
\text { tural assistance }\end{array}$ \\
\hline 2 & $\begin{array}{l}\text { Uses tactile screen with mini- } \\
\text { mal supervision }\end{array}$ & $\begin{array}{l}\text { Subject is able to drive the } \\
\text { wheelchair. Seeks support at } \\
\text { times }\end{array}$ \\
\hline 3 & $\begin{array}{l}\text { Uses tactile screen indepen- } \\
\text { dently and without supervision }\end{array}$ & $\begin{array}{l}\text { Subject drives the wheelchair } \\
\text { with ease and reaches the des- } \\
\text { tination without support }\end{array}$ \\
\hline
\end{tabular}

TABLE III

Rehabilitation Device Performance

\begin{tabular}{c|ccccc}
\hline & Control & \multicolumn{4}{c}{ Subjects } \\
& & $\min$ & $\max$ & mean & std \\
\hline Success & 1 & 1 & 1 & 1 & 0 \\
Path length (m) & 96 & 82 & 89.4 & 85.9 & 3.6 \\
Time (sec) & 717 & 935 & 2239 & 1408 & 572 \\
Mean velocity (m/sec) & 0.15 & 0 & 0.3 & 0.13 & 0.11 \\
\hline \# Collisions (system failure) & 0 & 0 & 2 & 0.75 & 0.95 \\
\# Collisions (inevitable) & 0 & 0 & 2 & 0.75 & 0.95 \\
\hline
\end{tabular}

$D_{1}$ ) and the ratio between far destinations minus arrow selections and the number of selections (denoted by $D_{2}$ ).

- Number of missions.

- Supervisor activity descriptor: measures the ratio between far destination pulsations and the total number of selections (denoted by $S_{A}$ ).

- Control activity descriptor: measures the ratio between missions aborted (pulsations on the stop icon) plus arrow pulsations, and the total number of selections (denoted by $\left.C_{A}\right)$.

Competence Analysis: A categorization of the subject competence was developed based on the feedback given by the subjects, teachers, and therapists (analysis based on the video recordings of the experimentation sessions and interviews). A protocol was defined for scores based on the operational and functional goals. An operational goal refers to the ease in which the subject uses the tactile screen. The functional goal refers to the ease in which the subject undergoes navigation during the experiments to reach the specified destination. The scores were tabulated in Table II.

\section{RESUlTS AND EVALUATION}

The overall result of the experiments showed that all subjects with cerebral palsy were able to carry out the navigation mission along the circuit. On the basis of these experiments, the results are analyzed and discussed in this section, according to the metrics defined in Section II-G-1. The performance of the intelligent wheelchair is discussed first, and then the behavior of the users to evaluate their adaptability to the rehabilitation device.

\section{A. Intelligent Wheelchair Performance Evaluation}

This section describes a general evaluation of the intelligent wheelchair and a particular evaluation of the two main systems: the human-machine interface (HMI) and the navigation technology. 

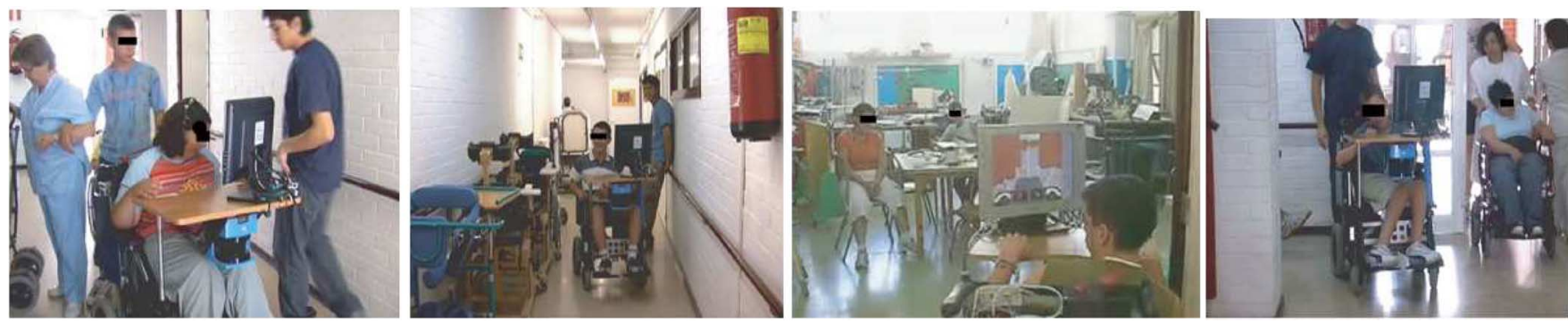

Fig. 5. Snapshots of the experimentation sessions at Alborada school. The trials were carried out during normal school working hours, making the scenario dynamic and unpredictable (there were students in motion, rehabilitation devices, furniture, etc.).

TABLE IV

USER INTERFACE PERFORMANCE

\begin{tabular}{c|ccccc}
\hline & Control & \multicolumn{4}{c}{ Subjects } \\
& & $\min$ & $\max$ & mean & std \\
\hline \# Total pulsations & 50 & 99 & 234 & 142.75 & 61.75 \\
\# Run icon pulsations & 20 & 31 & 77 & 48.75 & 19.77 \\
\# Stop icon pulsations & 7 & 18 & 39 & 28.25 & 10.30 \\
\# Right arrow pulsations & 1 & 4 & 14 & 6.50 & 5.25 \\
\# Left arrow pulsations & 1 & 8 & 35 & 17.50 & 12.76 \\
\hline \# Total goals & 21 & 26 & 69 & 41.75 & 18.83 \\
\# First grid row goals & 8 & 5 & 41 & 21.50 & 14.82 \\
\# Second grid row goals & 5 & 7 & 13 & 10.00 & 2.94 \\
\# Third grid row goals & 8 & 3 & 15 & 10.25 & 5.25 \\
\hline
\end{tabular}

1) Overall Performance: All subjects succeeded to navigate autonomously along the circuit (main task), which was a good indicator of the device utility. The results of the corresponding metrics are summarized in Table III. The path length and the execution time were very similar for all subjects, indicating a similar performance among them. The mean velocity of the wheelchair was $0.13 \mathrm{~m} / \mathrm{s}$. This is reasonable, given that the maximum speed was limited to $0.3 \mathrm{~m} / \mathrm{sec}$ for safety reasons, and that the wheelchair traversed narrow passages and stopped to avoid moving people. There were six collisions in all the experiments that required the intervention of the supervisor to select a command to free or unblock the wheelchair. Three of them were due to failures in the system, while three were due to the sensory capabilities of the robot. In the latter case, the robot collided with obstacles that were lower than the height of the laser, located $0.75 \mathrm{~m}$ above the ground. These results are very satisfactory as the experiments were carried out in working hours of the school and the scenario (Fig. 5) was not modified, which added a realistic but challenging difficulty component to the device since the scenario was very dynamic and in constant evolution.

2) User Interface Performance: Table IV summarizes the results of the user interface performance metrics. In general, the user interface performed well and all subjects were able to carry out the navigation task. Regarding the usability rate, in theory, with 2 or 3 pulsations (depending on whether the user stops the vehicle manually) it is possible to set a navigation mission. The real observed rate was 4.5 strokes per mission, as the mean of missions per experiment was 31.7 , resulting in a mean of 142.75 strokes to accomplish the main navigational task. This rate is acceptable as during the experiments there were many situations that increased the number of pulsations and missions. For example, the subjects used the arrows to orientate the vehicle instead of directly selecting a goal (which increased the number of missions), or they stopped the vehicle to change the goal when the trajectory was not the expected one. Furthermore, sometimes the subjects selected a goal, and before validating it, decided to select another goal (increasing the number of pulsations per mission). In addition, some children have a lower finger control and the pulsations were not accurate, producing multiple responses (see below).

The command utility was greater than zero for all subjects and commands, indicating that they used all the functionalities of the screen (no useless or extra commands were observed). The frequency of usage depended highly on the driving style, which will be analyzed in Section III-B. The errors in the user interface arose when the pulsations of the subjects were weak or when the finger slipped on the screen. In the first case, the stroke was not acquired, and in a very short period of time the stroke was repeated. In the second case, the consequence was an incorrect goal location. When the subjects perceived the situation they usually halted the vehicle. These errors are difficult to detect automatically, and were documented by visual inspection of the videos recorded during the experiments, preventing the provision of an automated quantitative evaluation.

3) Navigation Performance: In general, the performance of the navigation system was remarkable taking into account the difficulty imposed by the scenario. The experiments were carried out in a real scenario (a school) without modifying the environment or the daily activity. This includes the movement of people around the wheelchair and many situations with constrained space to maneuver, such as doorways or narrow passages around furniture or people (see Fig. 5). Table V shows the data for the navigation performance metrics. The system carried out 149 short-term missions, traveling a total of $343.7 \mathrm{~m}$ with a mean velocity of $0.13 \mathrm{~m} / \mathrm{s}$ (10 times less than the usual human walking speed). There were three collisions due to failures of the system; thus, the mission success was $97.98 \%$. Regarding the collision rates, there was a mean of 0.004 collisions per mission ( 1 collision every 50 missions), 0.86 collisions per $100 \mathrm{~m}$ ( 1 collision every $115 \mathrm{~m})$, and 0.025 collisions every $60 \mathrm{~s}$ (1 collision every $40 \mathrm{~min}$ ). In general, these are very low collision rates when considering a realistic application.

One of the main difficulties of current navigational systems is to avoid obstacles with safe margins and to drive the vehicle around obstacles in close proximity [36]. The minimum clearance and clearance means were 0.77 and $2.33 \mathrm{~m}$, which indicates that the vehicle carried out obstacle avoidance with good safety margins. The wheelchair was able to perform navigation in troublesome situations. For example, the mean of the narrower passage was $0.98 \mathrm{~m}$, which is very tight for the vehicle (the robot 
TABLE V

NAVIGATION PERFORMANCE

\begin{tabular}{c|ccccc}
\hline & Control & \multicolumn{4}{|c}{ Subjects } \\
& & $\min$ & $\max$ & mean & std \\
\hline \# Short-term missions & 22 & 26 & 36 & 31.7 & 4.3 \\
\hline Collisions / mission & 0 & 0 & 0.013 & 0.004 & 0.006 \\
Collisions / distance (100m) & 0 & 0 & 2.26 & 0.86 & 1.08 \\
Collisions / time (60sec) & 0 & 0 & 0.05 & 0.025 & 0.02 \\
\hline Clearance min & 0.9 & 0.74 & 0.75 & 0.77 & 0.73 \\
Clearance mean & 2.7 & 2.43 & 2.4 & 2.33 & 2.4 \\
\hline Shorter passage (m) & 1.10 & 0.8 & 1.11 & 0.98 & 0.01 \\
\# Passages width $<1 \mathrm{~m}$ & 0 & 0 & 3 & 0.7 & 0.9 \\
\# Passages width <1.5m & 12 & 28 & 40 & 33.5 & 5.9 \\
\hline
\end{tabular}

TABLE VI

TASK ACCOMPLISHMENT AND TIMES

\begin{tabular}{c|ccccc}
\hline & Control & \multicolumn{4}{|c}{ Subjects } \\
& & I & II & III & IV \\
\hline \# Missions & 22 & 26 & 31 & 34 & 36 \\
Path length (m) & 96 & 82 & 89.4 & 84 & 88.3 \\
Collisions (system/inevitable) & $0 / 0$ & $0 / 0$ & $0 / 0$ & $1 / 1$ & $2 / 2$ \\
\# of passages with width $<1.5$ & 12 & 28 & 29 & 40 & 37 \\
\hline \hline Total Time & 22 & 26 & 31 & 34 & 36 \\
Time moving & 96 & 82 & 89.4 & 84 & 88.3 \\
Time static & $0 / 0$ & $0 / 0$ & $0 / 0$ & $1 / 1$ & $2 / 2$ \\
\hline
\end{tabular}

size is $0.8 \times 1.2 \mathrm{~m}$, leaving only $0.1 \mathrm{~m}$ on each side); a mean of 0.7 and 33.5 of passages through widths inferior to 1 and $1.5 \mathrm{~m}$ was verified.

Finally, the integration between the previous systems is another issue for discussion. A typical metric used in this context is the integration delay [43], which is the time delay between the selection of an order in the user interface and its execution by the autonomous navigation system. This delay affects the awareness of the user about the state of the system. In this wheelchair, the integration was carried out so that the delay was confined to between zero and $0.2 \mathrm{~s} \mathrm{(inner} \mathrm{control} \mathrm{loop} \mathrm{period),} \mathrm{the} \mathrm{delay}$ being is very small and therefore not confusing the subjects.

\section{B. Users' Behavior Evaluation}

1) Execution Analysis: The results for the execution analysis metrics are summarized in Table VI.

The number of missions is the number of short-term missions needed to execute a complete navigational task. Subject I needed less missions than the other subjects, showing an efficient mission selection. Another predictor for the individuals subject navigation performance is the distance traveled. However, in this case this parameter is not as significant due to the small variability induced by the circuit (see Fig. 4). The execution times per subject provided very interesting results (see Table VI).

Subjects I, II, and III took comparatively less time than Subject IV. The time in motion was very similar for all subjects and the main difference was the time during which the wheelchair was not in motion, $T_{s}$, (when the user was selecting the next goal). Again Subject IV took more time than the others. Both observations can be understood based on the fact that Subject IV has a higher degree of cognitive disability, thereby taking more time to decide and accomplish a task.

The differences in the number of collisions among the different subjects is another measure of the navigation performance and of the cognitive abilities and adaptability for each individual. Subjects I and II had zero collisions and displayed better
TABLE VII

INTERACTION MODALITY METRICS

\begin{tabular}{l|ccccc}
\hline & Control & \multicolumn{4}{|c}{ Subjects } \\
& & I & II & III & IV \\
\hline$D_{1}=\frac{\text { \#Total Dest. }}{\text { \#Pulsations }}$ & 0.86 & 0.38 & -0.18 & 0.16 & -0.18 \\
$D_{2}=\frac{\text { \#Fr dest. -\#Arrows }}{\text { \#Pulsations }}$ & 0.2 & -0.04 & -0.17 & -0.22 & -0.28 \\
\# Missions & 22 & 26 & 31 & 34 & 36 \\
$S_{A}=\frac{\text { \#Far dest. }}{\text { \#Pulsations }}$ & 0.16 & 0.1 & 0.11 & 0.02 & 0.06 \\
$C_{A}=\frac{\text { \#Stops+\#Arrows }}{\text { \#Pulsations }}$ & 0.18 & 0.3 & 0.37 & 0.39 & 0.38 \\
\hline
\end{tabular}

navigation abilities than the other participants. This can also be correlated with the number of narrow passages encountered. Subjects I and II faced less number of narrow passages, probably due to a more efficient goal selection on the user machine interface.

Based on the previous parameters, it was possible to draw assumptions about the degree of adaptability of the system for the different subjects. The ease in which Subjects I, II, and III performed the task reflected a great adaptability to the device (autonomous wheelchair). The data (number of collisions, time during which the wheelchair was in motion and the time in which it was not) suggested that Subject IV had more difficulties in navigation, hence his interaction with the wheelchair may have been a result of a higher degree of cognitive impairment.

2) Activity Analysis: Table VII summarizes the results of the execution metrics. The activity discriminants $D_{1}$ and $D_{2}$, as well as the number of missions, are general metrics to differentiate between activity modes. The supervisory activity is characterized by a greater $D_{1}$ and $D_{2}$, and by a lower number of missions. The opposite is true for the control-oriented activity. Furthermore, the supervisory $\left(S_{A}\right)$ and control $\left(C_{A}\right)$ activity descriptors measure, respectively, the level of supervisory or control orientation required by the user.

From the tabular data it was evident that Subject I showed a higher tendency towards supervised activity. This can be understood in terms of higher values of $D_{1}, D_{2}$, lower number of missions, and a high $S_{A}$. Subject IV had a tendency towards control activity, since he had the lowest values of $D_{1}, D_{2}$, the maximum number of missions, and a low value of $C_{A}$. A gradual shift from supervisory activity to control activity was observed in Subjects II and III.

Subjects I and II, who showed a supervisory oriented activity, also showed a better navigation performance, according to the metrics of the execution analysis (see Table VI). Subjects III and IV showed different levels of intervention in the wheelchair control; in general, they were less inclined to let the wheelchair reach the intermediate goals, which led to the lowest execution performance (see Table VI). This indicates that a supervisory-oriented activity explores the facilities of the system best for autonomous navigation, resulting in a more efficient performance.

Based on the previous analysis, the above metrics gave an indication on the level of system awareness and on the mental model development between the two modes of activity for subjects with different profiles of cognitive disability. The adherence towards control activity in Subject IV, in terms of the dif- 
TABLE VIII

SUMMARY OF COMPETENCE MEASURES

\begin{tabular}{|c|c|c|c|c|}
\hline$\overline{\text { Measurements }}$ & Subject I & Subject II & Subject III & Subject IV \\
\hline $\begin{array}{l}\text { Functional Goal } \\
\text { Attainment } \\
\text { [Initial/Final] }\end{array}$ & $\begin{array}{l}\text { Better maneuverability and } \\
\text { anticipation of turns. }[2 / 3]\end{array}$ & $\begin{array}{l}\text { Seeks supervision at times. } \\
\text { Anticipates turns. Seeks } \\
\text { hand gestures from the ed- } \\
\text { ucators. [2/3] }\end{array}$ & $\begin{array}{l}\text { The instructor has to as- } \\
\text { sist especially when making } \\
\text { sharp turns and complex nav- } \\
\text { igational tasks. }[2 / 3]\end{array}$ & $\begin{array}{l}\text { Needs continual support during ex- } \\
\text { perimentation. }[1 / 2]\end{array}$ \\
\hline $\begin{array}{l}\text { Operational } \\
\text { Goal Attainment } \\
\text { [Initial/Final] }\end{array}$ & $\begin{array}{l}\text { Shows interest in using the } \\
\text { tactile screen. Can locate tar- } \\
\text { gets and execute tasks with } \\
\text { minimal supervision. }[2 / 3]\end{array}$ & $\begin{array}{l}\text { Overcame initial problems } \\
\text { with the tactile screen. In- } \\
\text { creased interest with expe- } \\
\text { rience in navigation. }[1 / 3]\end{array}$ & $\begin{array}{l}\text { Difficulty in understanding } \\
\text { the association between goal } \\
\text { locations and the points on } \\
\text { the tactile screen. }[1 / 3]\end{array}$ & $\begin{array}{l}\text { Difficulties in getting familiarized } \\
\text { with the tactile screen. Shows dis- } \\
\text { appointment when not able to per- } \\
\text { form the task correctly. }[1 / 2]\end{array}$ \\
\hline $\begin{array}{l}\text { Teachers and thera- } \\
\text { pists assessment }\end{array}$ & $\begin{array}{l}\text { Enthusiastic about driving } \\
\text { the wheelchair. Shows better } \\
\text { anticipation. Cheerful during } \\
\text { experimentation. }\end{array}$ & $\begin{array}{l}\text { Good receptivity, limited } \\
\text { expressive language; indi- } \\
\text { cates likes and dislikes, } \\
\text { vocalizations (few words). }\end{array}$ & $\begin{array}{l}\text { Initially withdrawn. } \\
\text { Interested in driving the } \\
\text { wheelchair. }\end{array}$ & $\begin{array}{l}\text { Minimal vocalizations. Shows un- } \\
\text { happiness with failures. Needs con- } \\
\text { stant encouragement during the } \\
\text { task. }\end{array}$ \\
\hline
\end{tabular}

ficulty to create a mental model, seems to be the a result of his greater cognitive impairment. The other subjects with comparatively higher cognitive capabilities showed more effective mental models and an interaction mode closer to a supervisory role.

3) Competence Analysis: The scores defined in Table II were used as the basis for a competence assessment. Table VIII shows the scores for functional goal attainment, operational goal attainment, and subjective assessment. The evaluation is based on the feedback of teachers, parents, and therapists, as well as on the interviews with the subjects during and after the experiment sessions.

From the functional goal attainment perspective, Subjects I, II, and III showed similar performance scores [2/3]. The improvement in the initial score of 2 to the final functional goal score of 3 indicated a learning behavior during the navigational task. The subjects were able to drive the wheelchair to the specified destination with ease, requiring occasional supervision. Subject IV sought continual supervision but eventually was successful in accomplishing the navigational task. From the operational goal point of view, all subjects were able to choose goal locations with ease and to operate the tactile screen to drive the wheelchair; however, Subject IV needed more training and continual supervision as was evident from his low score [1/2]. Based on the subjective feedback given to teachers and therapists, it was concluded that all subjects showed keen interest in driving the wheelchair using the tactile screen and were enthusiastic about avoiding obstacles, anticipating turns, and finally reaching the destination.

\section{CONCLUSION}

An intelligent robotic wheelchair adapted for subjects with cognitive disabilities due to Cerebral palsy was presented. The results of the field study suggested that, after a short training phase, the subjects were able to drive this type of system, even in situations where navigation was difficult. The key of the success of the system was the design and combination of state of the art navigation technologies and user interfaces engineering. The navigation technologies provided safe and reliable navigation capabilities even in unknown and dynamic scenarios, while user interface allowed the children to intuitively interact with the wheelchair.
Based on qualitative and quantitative measures recorded during the field trials, a technical evaluation was carried out, on the performance of the system and the on the behavior of the subjects. The results gave indications on the mental model created by the subjects, pointed out a weakness in the design (in terms of usability), and provided valuable feedback to improve the design based on the personal needs and preferences of the subjects. The authors are well aware of the fact that the number of subjects in the study is small; however, the consistent behavior of both the system and users with different degrees of cognitive disabilities provides strong evidence that they are able to drive the vehicle to specified destinations by appropriately selecting target locations, to perform complex maneuverability tasks, and to understand the system. Furthermore, their performance in terms of total time and distance, or number of commands, was in the same order of magnitude as the control subject.

In summary, this autonomous navigation based wheelchair system shows prospects for a wide range of rehabilitation benefits for users with cognitive disabilities, and in particular, for users with cerebral palsy. In addition to more extensive tests, the authors are currently working on the improvement of design and training procedures, to create a better mental model of the vehicle, and on the incorporation of adaptive strategies to modify the interface during the operation.

\section{ACKNOWLEDGMENT}

The authors would like to thank the Colegio Público de Educación Especial Alborada, Zaragoza for the support, and especially the educators and therapists J. Pegueiro, J. Manuel Marcos, and C. Canalís for their help, comments, and work with the children during the project. The author would like to thank to the children and parents for their cooperation, which is invaluable in the ongoing research.

\section{REFERENCES}

[1] M. Jones and J. Sanford, "People with mobility impairments in the united states today and in 2010," Assistive Technol., vol. 8, pp. 43-53, 1996.

[2] L. Fehr, W. Langbein, and S. Skaar, "Adecuacy of power wheelchair control interfaces for persons with severe disabilities: A clinical survey," J. Rehabil. Res. Develop., pp. 353-360, 2000.

[3] R. Simpson, "Smart wheelchairs: A literature overview," Int. J. Rehabil. Res., vol. 42, no. 4, pp. 423-436, 2005. 
[4] I. A. Adelola, S. L. Cox, and A. Rahman, "Adaptable virtual reality interface for powered wheelchair training of disabled children," in Proc. 4th Int. Conf. Disability, Virtual Reality Assoc. Tech., 2002.

[5] R. Simpson, E. LoPresti, and R. Cooper, "How many people would benefit from a smart wheelchair? Journal of rehabilitation research and development," Arch. Phys. Med. Rehabil., vol. 45, no. 1, pp. 53-72, 2008.

[6] V. Kumar, T. Rahman, and V. Krovi, "Assistive devices for people with motor disabilities," in Wiley Encyclopedia for Electrical and Electronic Engineers. New York: Wiley, 1997.

[7] H. A. Yanco, "Integrating robotic research: A survey of robotic wheelchair development," in AAAI Spring Symp. Integrating Robot. Res., 1998.

[8] D. Ding and R. Cooper, "Electric-powered wheelchairs: A review of current technology and insight into future directions," IEEE Control Syst. Mag., vol. 25, no. 2, pp. 22-34, 2005.

[9] D. Mestre, J. Pergandi, and P. Mallet, "Virtual reality as a tool for the development of a smart wheelchair," in Symp. Laval Virtual 2006, France, 2006.

[10] L. Montesano, J. Minguez, J. Alcubierre, and L. Montano, "Towards the adaptation of a robotic wheelchair for cognitive disabled children," in IEEE Int. Conf. Intell. Robot Syst., Beijin, China, 2006, pp. 710-716.

[11] L. Conway, R. Volz, and M. Walker, "Teleautonomous systems: Projecting and coordinating intelligent action at a distance," IEEE Trans. Robot. Autom., vol. 6, no. 2, pp. 146-158, Apr. 1990.

[12] J. Crisman, M. Cleary, and J. Rojas, "The deictically controlled wheelchair," Image Vis. Comput., vol. 16, pp. 235-249, 1998.

[13] G. Bourhis, O. Horn, and O. H. A. Pruski, "An autonomous vehicle for people with motor disabilities," IEEE Robot. Autom. Mag., vol. 8, no. 1, pp. 20-28, Mar. 2001.

[14] A. Lankenau and T. Rofer, "A versatile and safe mobility assistant," IEEE Robot. Autom. Mag., vol. 8, no. 1, pp. 29-37, Mar. 2001.

[15] E. Prassler, J. Scholz, and P. Fiorini, "A robotic wheelchair for crowded public environments," IEEE Robot. Autom. Mag., vol. 8, no. 1, pp. 38-44, Mar. 2001.

[16] S. P. Levine, D. A. Bell, L. A. Jaros, R. C. Simpson, Y. Koren, and J. Borenstein, "The navchair assistive wheelchair navigation system," IEEE Trans. Rehabil. Eng., vol. 4, no. 4, pp. 443-451, Dec. 1999.

[17] J. Pineau, "Smartwheeler: A robotic wheelchair test-bed for investigating new models of human-robot interaction," in Assoc. Adv. Artif. Intell. Spring Symp., 2007.

[18] J. d. M. J. Philips, G. Vanacker, E. L. F. Galán, P. Ferrez, H. V. Brussel, and M. Nuttin, "Adaptive shared control of brain actuated simulated wheelchair," in IEEE Int. Conf. Rehabil. Robot., 2007, pp. 408-414.

[19] Q. Zeng, E. Burdet, B. Rebsamen, and C. L. Teo, "A collaborative wheelchair system," IEEE Trans. Neural Syst. Rehabil., vol. 16, no. 2, pp. 161-170, Apr. 2008.

[20] K. T. Tsui and H. Yanco, "Simplifying wheelchair mounted robotic arm control with a visual interface," in AAAI-Spring Symp., 2007.

[21] T. Adlam, "Technology, autonomy and cognitive disability" in UbiHealth 2003: 2nd Int. Workshop Ubiquitous Comput. Pervasive Healthcare Appl., 2003.

[22] M. Mazo, "An integral system for assisted mobility," IEEE Robot. Autom. Mag., vol. 8, no. 1, pp. 46-56, Mar. 2001.

[23] C. Martens, N. Ruchel, O. Lang, O. Ivlev, and A. Graser, "A friend for assisting handicapped people," IEEE Robot. Autom. Mag., vol. 8, no. 1, pp. 57-65, Mar. 2001.

[24] H. A. Yanco, Wheelesley, A Robotic Wheelchair System: Indoor Navigation and User Interfaz, V. Mittal, H. Yanco, J. Aronis, and R. Simpson, Eds. New York: Springer, 1998, pp. 256-268.

[25] I. Iturrate, J. Antelis, A. Kübler, and J. Minguez, "Non-invasive brainactuated wheelchair based on a 300 neurophysiological protocol and automated navigation," IEEE Trans. Robot., submitted for publication.
[26] Y. Kuno, N. Shimada, and Y. Shirai, "Look where you are going," IEEE Robot. Autom. Mag., vol. 10, no. 1, pp. 21-34, Mar. 2003.

[27] K. Tsui, H. Yanco, D. Kontak, and L. Beliveau, "Development and evaluation of a flexible interface for a wheelchair mounted robotic arm," presented at the Proc. 3rd Annu. ACM/IEEE Conf. Human-Robot Interaction, Amsterdam, The Netherlands, 2008

[28] F. D. Rose, B. M. Brooks, and A. A. Rizzo, "Virtual reality in brain damage rehabilitation: Review," CyberPsychol. Behavior, vol. 8, no. 3, pp. 241-262, 2005.

[29] M. Schultheis and R. Mourant, "Virtual reality and driving: The road to better assessment for cognitively impaired populations," Presence: Teleoperators Virtual Environ., vol. 10, no. 4, pp. 431-439, 2001.

[30] D. Spaeth, H. Mahajan, A. Karmarkar, D. Collins, R. Cooper, and M. Boninger, "Development of a wheelchair virtual driving environment: Trials with subjects with traumatic brain injury," Arch. Phys. Med. Rehabil., vol. 89, no. 5, pp. 996-1003, 2008.

[31] M. Endsley, "Design and evaluation for situation awareness enhacement," in Human Factors Soc. 32nd Annu. Meeting, 1988, pp. 97-101.

[32] J. Scholtz, "Human-robot interactions: Creating synergistic cyber forces," in NRL Workshop Multirobot Syst., 2002.

[33] R. Meier, T. Fong, C. Thorpe, and C. Baur, "A sensor fusion based user interface for vehicle teleoperation," in Int. Conf. Field Service Robot, 1999.

[34] T. Fong, C. Thorpe, and C. Baur, "A sensor fusion based user teleoperation: Collaborative control, sensor fusion displays, and remote driving tools," Autonomous Robots, vol. 11, no. 1, pp. 77-85, 2001.

[35] C. Erren-Wolters, H. van Dijk, A. de Kort, M. IJzerman, and M. Jannink, "Virtual reality for mobility devices: Training applications and clinical results: A review," Int. J. Rehabil. Res., vol. 30, no. 2, pp. 91-96, 2007.

[36] L. Montesano, J. Minguez, and L. Montano, "Lessons learned in integration for sensor-based robot navigation systems," Int. J. Adv. Robot. Syst., vol. 3, no. 1, pp. 85-91, 2006.

[37] J. Minguez and L. Montano, "Sensor-based robot motion generation in unknown, dynamic and troublesome scenarios," Robot. Auton. Syst., vol. 52, no. 4, pp. 290-311, 2005.

[38] L. Montesano, J. Minguez, and L. Montano, "Modeling dynamic scenarios for local sensor-based motion planning," Autonomous Robots, vol. 25 , no. 3, pp. 231-251, 2008.

[39] A. Ranganathan and S. Koenig, "A reactive architecture with planning on demand," in Int. Con. Robot. Autom., Las Vegas, NV, 2003, pp. $1462-1468$.

[40] J. Minguez and L. Montano, "Nearness Diagram (ND) navigation: Collision avoidance in troublesome scenarios," IEEE Trans. Robot. Autom., vol. 20, no. 1, pp. 45-59, Feb. 2004.

[41] G. Silke-Birgitta, "Research in interpersonal violence-A constant balancing act," in The Role of the Researcher in Qualitative Psychology, M. Kiegelmann, Ed. : , 2002, pp. 159-168.

[42] R. Simpson, D. Poirot, and M. F. Baxter, "Evaluation of the hephaestus smart wheelchair system," presented at the Int. Conf. Rehabil. Robot., Stanford, CA, 1999.

[43] B. Kuipers, Building and Evaluating an Intelligent Wheelchair 2006, Internal Report.

[44] P. Holliday, A. Mihailidis, R. Rolfson, and G. Fernie, "Understanding and measuring powered wheelchair mobility and manoeuvrability. Part I. Reach in confined spaces," Disabil. Rehabil., vol. 27, pp. 939-949, 2005.

[45] H. A. Yanco and J. Drury, "Classifying human-robot interaction: An updated taxonomy," in IEEE Int. Conf. Syst., Man Cybern., The Hague, The Netherlands, Oct. 2004, vol. 3, pp. 2841-2846.

Authors' photographs and biographies not available at the time of publication. 